\title{
Miosite ossificante em músculo temporal: relato de caso
}

\author{
Myositis ossificans in temporal muscle: case report
}

\author{
Paulo Ribeiro de Queiroz Neto* \\ Bartolomeu Conceição Bastos Neto** \\ Christiano Sampaio Queiroz ${ }^{* * *}$
}

\section{Resumo}

A miosite ossificante é uma desordem não neoplásica, caracterizada por formação óssea ectópica dentro do músculo ou de sua fáscia. Pode ser definida como um processo progressivo ou de origem traumática. Quando acomete os músculos da mastigação, leva a uma diminuição progressiva da abertura bucal. Radiograficamente, essas lesões são bem circunscritas. Objetivo: apresentar um relato de caso clínico de miosite ossificante em músculo temporal. Relato de caso: um paciente do gênero masculino, 15 anos, vítima de acidente motociclístico, compareceu ao Hospital de Urgências e Emergências do Subúrbio, Salvador, BA, cursando com fratura de complexo zigomático direito e processo coronoide mandibular direito. Então, foi submetido à osteossíntese do complexo zigomático e encaminhado para acompanhamento ambulatorial. No segundo mês pós-operatório, evoluiu com uma limitação de abertura bucal, quando foi observada, em exame de imagem, uma formação óssea ectópica em região de incisura sigmoide direita. Optou-se por nova intervenção cirúrgica com exérese da neoformação óssea, utilizando o acesso intraoral de Obwergeiser e exposição do processo coronoide, região medial e lateral do ramo mandibular direito. Realizou-se osteotomia do coronoide com remoção da massa óssea ectópica. Considerações finais: o tratamento da miosite ossificante envolvendo os músculos da mastigação ainda é controverso e exige uma larga experiência do cirurgião.

Palavras-chave: Odontologia. Trauma. Traumatismos faciais.

\section{Introdução}

A calcificação muscular é uma desordem não neoplásica, caracterizada por formação óssea ectópica dentro do músculo ou de sua fáscia. Pode ser definida como uma desordem progressiva, autossômica dominante ou de origem traumática, com múltiplos sítios de formação óssea em musculatura, fáscias, tendões e ligamentos ${ }^{1-3}$.

Calcificação muscular traumática, também denominada circunscrita, é um defeito originado de trauma agudo, queimaduras, manipulação cirúrgica ou injúrias repetitivas. Diferentemente da variante progressiva, a remoção cirúrgica é bem efetiva, embora algumas recorrências possam ocorrer ${ }^{3}$. Alguns autores têm discordado da nomenclatura traumática, pois, em alguns casos, uma história de trauma não pode ser relacionada. Sugerem-se, então, outros termos, como ossificação heterotópica, tumor ósseo pseudomaligno dos tecidos moles e fibrodisplasia ossificante circunscrita ${ }^{1}$.

A miosite ossificante dos músculos da mastigação não é observada com frequência na literatura, sendo seu achado clínico mais comum a limitação de abertura bucal. Sua patogênese ainda permanece incerta, de modo que várias teorias foram propostas. A mais aceita seria a diferenciação de células extraósseas por meio do estímulo de proteínas morfogenéticas, lançadas a partir de fontes de osso nativo após uma lesão ou trauma ${ }^{3}$.

Essa desordem é descrita como um processo reparativo benigno de ossificação ectópica nos tecidos moles. Várias causas levam ao acometimento dos músculos da mastigação, dentre elas, estão: extração dentária, disseminação de abscessos odontogênicos, anestesia local, uso de colar cervical, mento-

Cirurgião bucomaxilofacial pela Universidade Federal da Bahia (Ufba), professor de anotomia humana, Faculdade Maria Milza (Famam), Cruz das Almas, Bahia, Brasil.

Graduando em Odontologia, Famam, Cruz das Almas, Bahia, Brasil.

Doutor e mestre em Odontologia pela Ufba, professor do curso de Odontologia da Faculdade Ruy Barbosa, Salvador, Bahia, Brasil. 
plastia, tratamento ortodôntico mal realizado, traumas diretos e fraturas do esqueleto ${ }^{4}$.

Tendo em vista o exposto, o presente trabalho tem por objetivo apresentar um relato de caso clínico de miosite ossificante em músculo temporal, ocorrida após fratura e correção cirúrgica de complexo zigomático.

\section{Relato de caso}

Um paciente do gênero masculino (Figura 1), de 15 anos de idade, compareceu ao Hospital de Urgências e Emergências do Subúrbio, Salvador, BA, vítima de acidente motociclístico, apresentando fratura de complexo zigomático direito e processo coronoide mandibular direito. Então, foi submetido à osteossíntese do complexo zigomático e encaminhado para acompanhamento ambulatorial. No segundo mês pós-operatório, evoluiu com uma limitação de abertura bucal progressiva, na qual foi observada, em exame de imagem pós-operatório, formação óssea ectópica em região de incisura sigmoide direita. Durante a anamnese, o paciente não referiu patologias de base ou alterações sistêmicas. No exame físico, notou-se limitação de abertura bucal e boa projeção do osso zigomático direito (Figuras 2 e 3). Pelo exame de imagem pré-operatório, nota-se material de síntese óssea em posição e massa óssea neoformada ectópica em região do músculo temporal.

Optou-se, então, por nova intervenção cirúrgica, com exérese da neoformação óssea, utilizando o acesso intraoral de Obwergeiser e exposição do processo coronoide, região medial e lateral do ramo mandibular direito. Realizou-se osteotomia do coronoide com remoção da massa óssea ectópica do lado acometido (Figuras 4 e 5). No transoperatório, notou-se melhora acentuada na abertura bucal (Figura 6). Em exame de imagem pós-operatório de sete dias, notou-se remoção completa do coronoide e da massa óssea ectópica.

Como orientações pós-operatórias, foi instituída fisioterapia precoce por meio de exercícios com espátulas de madeira e fonoterapia, realizados duas vezes ao dia, e a interposição de espátulas era sessada a um grande estímulo doloroso; aguardavam-se então 10 minutos para remoção do dispositivo. Realizou-se, também, prescrição de terapia com anti-inflamatório e antibiótico. O paciente evoluiu em pós-operatório sem intercorrências, sendo relatada discreta diminuição do nível de abertura bucal no exame pós-operatório de seis meses (Figura 7). $\mathrm{O}$ paciente relatou diminuição da frequência dos exercícios fisioterápicos devido à sintomatologia dolorosa associada.

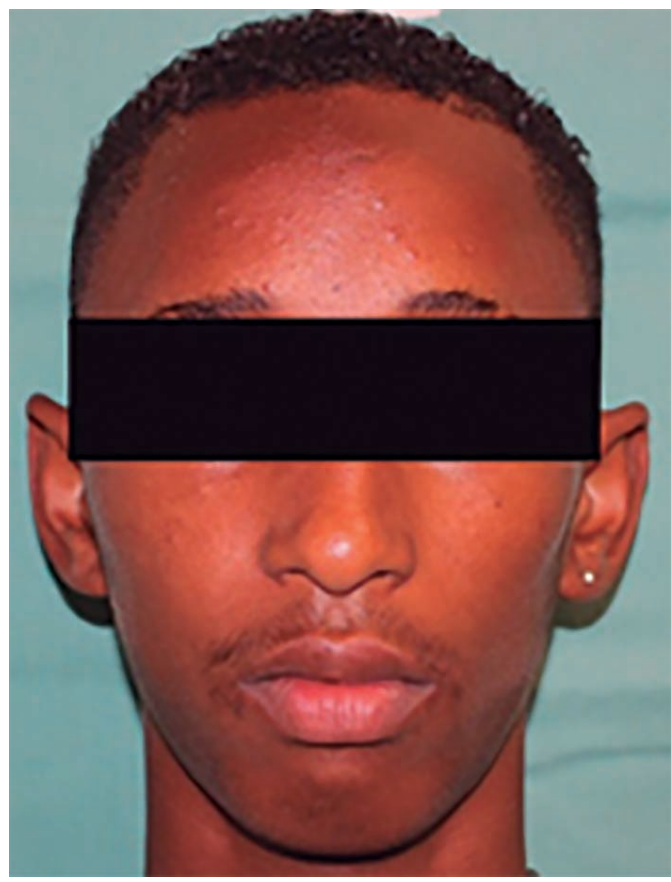

Figura 1 - Foto frontal

Fonte: elaboração dos autores.

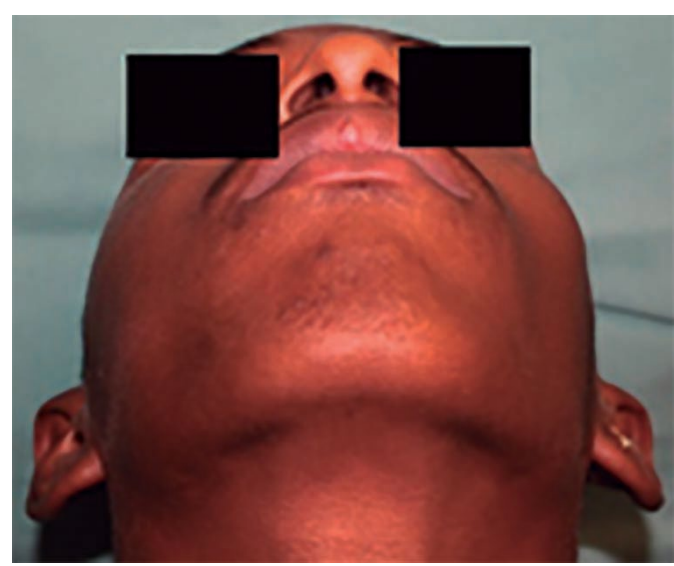

Figura 2 - Foto caudocraniana - demonstrando boa projeção anteroposterior do zigoma

Fonte: elaboração dos autores.

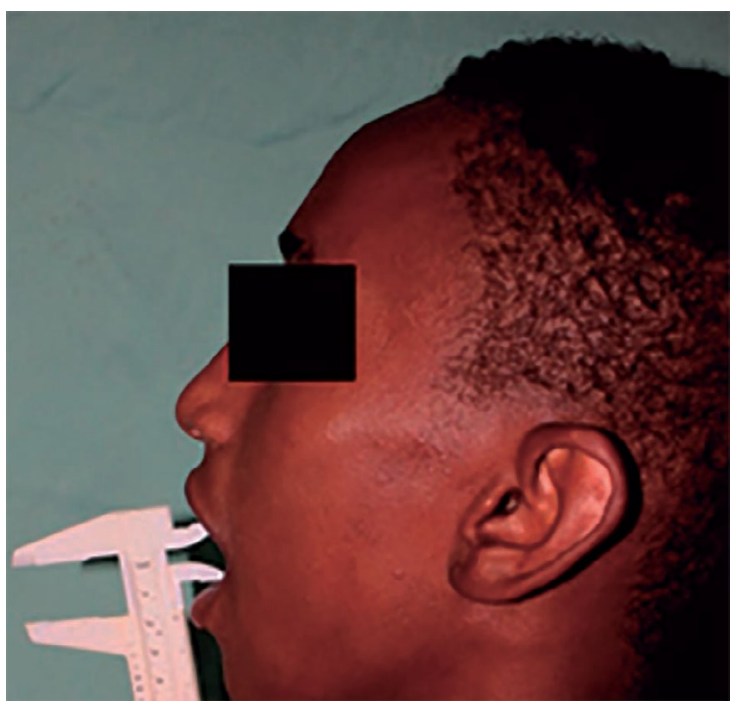

Figura 3 - Abertura inicial de $17 \mathrm{~mm}$

Fonte: elaboração dos autores. 


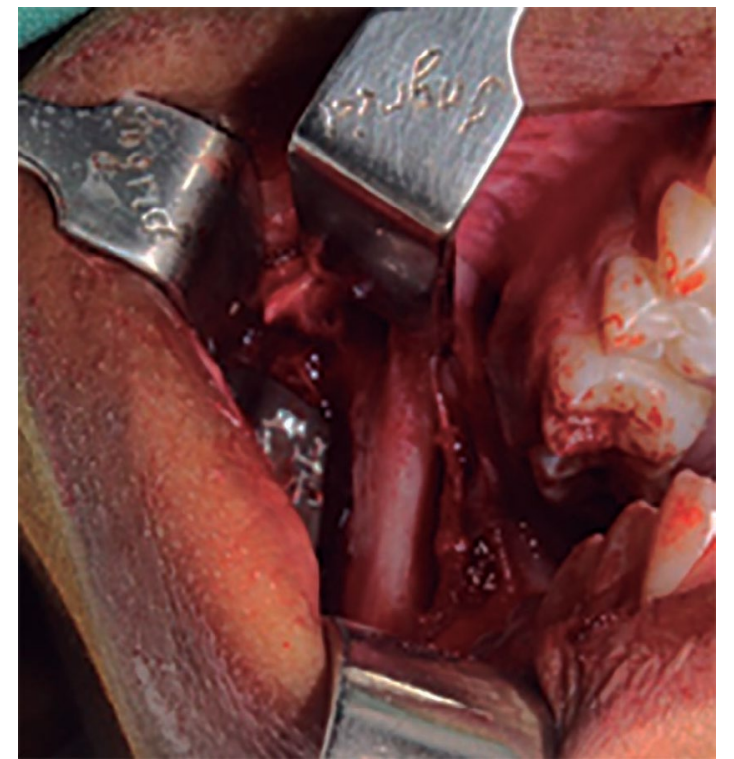

Figura 4 - Aspecto transoperatório - loja cirúrgica

Fonte: elaboração dos autores.

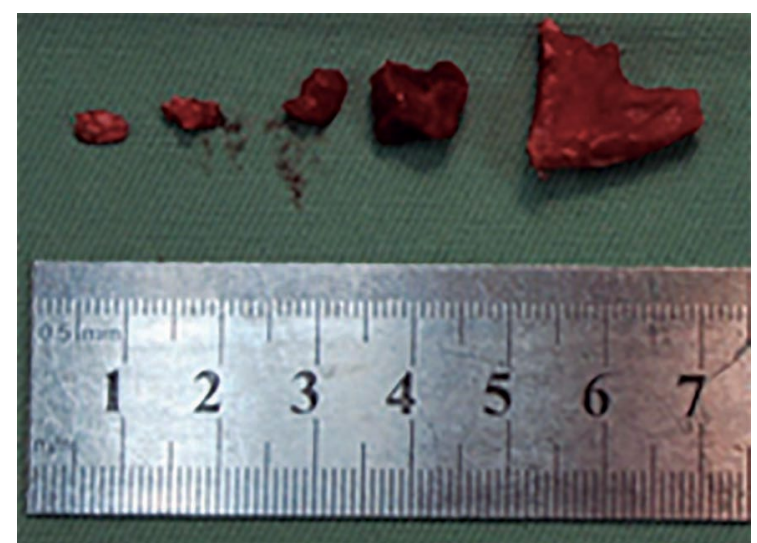

Figura 5-Peça Cirúrgica

Fonte: elaboração dos autores.

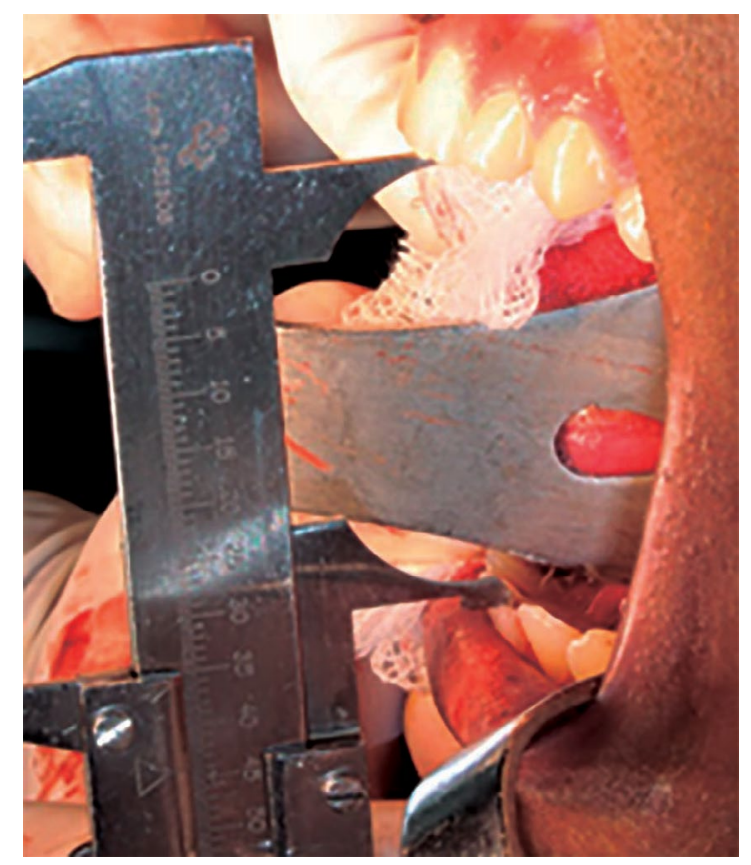

Figura 6 - Abertura bucal - transoperatório de $45 \mathrm{~mm}$

Fonte: elaboração dos autores.

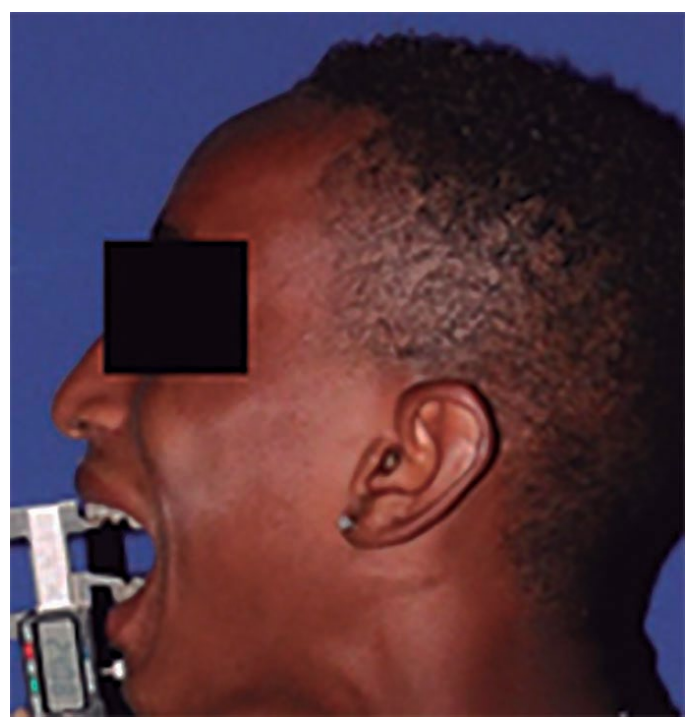

Figura 7 - Abertura bucal - 4 meses - 22,8 mm

Fonte: elaboração dos autores.

\section{Discussão}

No paciente acompanhado pelo estudo, observa-se que o músculo temporal foi afetado. Esse achado corrobora os resultados encontrados por Boffano et al. ${ }^{3}$ (2013), que relataram um caso sobre a calcificação ectópica dos músculos da mastigação. Os autores observaram que o músculo masseter era o mais frequentemente envolvido, seguido do pterigoide medial, lateral e temporal. A alta incidência do músculo masseter foi relacionada à sua posição mais externa, que favorece o trauma.

O diagnóstico da miosite ossificante pode ser desafiante, e uma anamnese acurada é essencial. Histórico de trauma e procedimentos cirúrgicos prévios podem levar, juntamente com um quadro de limitação de abertura bucal, ao descobrimento da enfermidade. Exames de imagem auxiliam no diagnóstico e no planejamento cirúrgico, enquanto a tomografia computadorizada é fundamental para o correto tratamento, avaliando quantidade e sítio da ossificação $0^{4,5}$. No caso clínico relatado, o paciente diagnosticado com miosite ossificante relatou ter sofrido um acidente motociclístico e sentir limitação progressiva de abertura bucal. Os exames de imagem mostraram que havia uma neoformação óssea na região do músculo temporal.

Radiograficamente, essas lesões são bem circunscritas, tendo uma maior radiopacidade na periferia, podem ou não estar relacionadas ao osso adjacente, caracterizando-se como uma calcificação amorfa dentro dos tecidos moles ${ }^{6}$.

Realizar o diagnóstico diferencial de outras patologias que causam a limitação de abertura bucal (anquilose, degeneração do disco articular, luxação anterior do disco sem redução) pode se tornar um desafio. A suspeita clínica deve ser confirmada e suportada por exames complementares de imagem ${ }^{3,5}$. 
Histologicamente, as miosites ossificantes são formadas por osso lamelar maduro e osteoblastos ativos na periferia, zona intermediária composta por osteoide, cartilagem e uma área central formada por proliferação fibroblástica, células fusiformes e células mesenquimais progenitoras ${ }^{6}$.

Dentre as formas de tratamento descritas na literatura, a excisão cirúrgica da massa calcificada foi a predominante ${ }^{1,3,7}$. Alguns cirurgiões optam por incluir mais um procedimento cirúrgico, como: a remoção do músculo por completo, uma coronoidectomia e/ou condilectomia, ou a interposição de enxerto de gordura e alguns materiais aloplásticos. Outros apenas executam biopsia e sugerem tratamento fisioterápico intenso por meio de exercícios $^{4,7,8}$. Todavia, o tratamento é controverso e pode variar conforme a experiência do cirurgião. No caso apresentado, o tratamento instituído foi a intervenção cirúrgica com a remoção da massa óssea do lado acometido. Não existe um protocolo de tratamento definido ${ }^{1,9}$.

O objetivo do tratamento foi alcançado, uma vez que não houve recidiva da enfermidade e restabeleceu-se a abertura bucal do paciente de forma funcional e satisfatória.

\section{Considerações finais}

O tratamento da miosite ossificante envolvendo os músculos da mastigação ainda é controverso e exige uma larga experiência do cirurgião. Seu alto potencial de recidiva e sua etiopatogenia desconhecida dificultam o tratamento. A colaboração do paciente e a implantação de fisioterapia precoce melhoram e otimizam os resultados clínicos. São necessários mais estudos e comparação para se definir a melhor conduta terapêutica a ser empregada.

\section{Abstract}

Ossific myositis is a non-neoplastic disorder, characterized by ectopic bone formation within the muscle or its fascia. It can be defined as a progressive or traumatic process. When it affects the chewing muscles, it leads to a progressive decrease of the mouth opening. Radiographically, these lesions are well circumscribed. Objective: the objective of this study was to present a case report of ossificatory myositis in the temporal muscle. Case Report: a 15-year-old male patient, a motorcycle accident victim, attended the Suburb Emergency and Emergency Hospital, Salvador, Bahia, attending a fracture of the right zygomatic complex and a right mandibular coronoid process. He was submitted to osteosynthesis of the zygomatic complex and referred for outpatient follow-up. In the second postoperative month, there was a limitation of oral opening, in which an ectopic bone formation in the right sigmoid incision region was observed in the imaging examination. We opted for a new surgical intervention with exérese of the new bone formation, using the intraoral access of Obwergeiser and exposure of the coronoid process, medial and lateral region of the right mandibular branch. Coronary osteotomy was perfor- med with removal of ectopic bone mass. Final considerations: the treatment of ossificating myositis involving mastication muscles is still controversial and requires a great deal of experience from the surgeon.

Keywords: Dentistry. Trauma. Facial trauma.

\section{Referências}

1. Kim DD, Lazou SK, El GH, Berger JR. A case report and literature review. J Oral Maxillofac Surgery 2002; 60(3):1072-6.

2. Aoki T, Naito H, Ota Y, Shhiiki K. Myositis ossificans traumatica of the masticatory muscles: Review of the literature and report of a case. J Oral Maxillofac Surgery 2002; 60(9):1083-8.

3. Boffano P, Zavattero E, Bosco G, Berrone S. Myositis ossificans of the left medial pterygoid muscle: case report and review of the literature of myositis ossificans of masticatory muscles. Craniomaxillofacial Trauma and Reconstruction 2013; 7(1):43-50.

4. Spinzia A, Moscato G, Broccardo E, Castelletti, Maglitto F, Orabona GDA, et al. A rare isolated unilateral myositis ossificans traumatica of the lateral pterygoid muscle: a case report. J Medical Case Reports 2014; 8(1):1-5.

5. Nemoto H, Sumiya N, Ito Y, Kimura N, Akizuki A, Maruyama N. Myositis Ossificans Traumatica of the Masticatory Muscles. J Craniofac Surgery 2012; 23(5):514-6.

6. Torres AM, Nardis AC, da Silva RA, Savioli C. Myositis ossificans traumatica of the medial pterygoid muscle following a third molar extraction. Int J Oral Maxillofac Surgery 2014; 44(4):488-90.

7. Rattan V, Rai S, Vaiphei K. Use of buccal pad of fat to prevent heterotopic bone formation after excision of myositis ossificans of medial pterygoid muscle. J Oral Maxillofac Surgery 2008; 66(7):1518-22.

8. Mevio E, Rizzi L, Bernasconi G. Myositis ossificans traumatica of the temporal muscle: a case report. Auris Nasus Larynx 2001; 28(4):345-7.

9. Schiff MJ, Meara DJ. Myositis ossificans of the temporalis muscle: case report and review of the literature. J Oral Maxillofac Surgery 2013; 71(11):1893-8.

\section{Endereço para correspondência:}

Paulo Ribeiro de Queiroz Neto

Rua Esplanada, 222, Centro

CEP: 44190-000, Santo Estevão, Bahia, Brasil

Telefone: (75) 992284220

E-mail: bbastosneto@hotmail.com

Recebido: 04/09/17. Aceito: 20/11/17. 